\title{
Estado de conservación y distribución del pez de agua dulce Capitán de la Sabana Eremophilus mutisii en Cundinamarca, Colombia
}

\author{
Conservation status and distribution of the freshwater Catfish Eremophilus mutisii \\ (Trichomycteridae) in Cundinamarca, Colombia
}

\begin{abstract}
Ciromar Lemus-Portillo ${ }^{1 *}$, Monika C. Echavarria-Pedraza ${ }^{1 *}$, Jhon E. Rojas ${ }^{2}$, Jimmy E. Alvarez-Diaz ${ }^{1}$, Kelly J. León-Pardo ${ }^{1}$, K. Samantha Aguilar-Orjuela ${ }^{1}$, Jhonathan F. Maldonado ${ }^{1}$
\end{abstract}

\section{Resumen}

Eremophilus mutisii (Humboldt, 1805) es un pez endémico del altiplano cundiboyacense, cuya categoría de amenaza actual es vulnerable. El programa nacional para la conservación de esta especie sirvió de base para el plan de conservación en la jurisdicción CAR Cundinamarca de 2018. Este plan se sustentó en el diagnóstico del estado de conservación de la especie presentado en este estudio. Para ello, se investigó su distribución geográfica a partir de colecciones biológicas y salidas de campo a ecosistemas acuáticos previamente identificados por la CAR y el grupo investigador. Se evaluó la presencia de $E$. mutisii en los ríos Suárez, Bogotá (cuenca alta), Ubaté, Veraguas, Frío, Aguas Claras y Muña, y en la laguna de Fúquene y los embalses del Neusa y Tominé, mediante un protocolo de muestreo basado en la pesca eléctrica y la toma de parámetros fisicoquímicos. El esfuerzo de pesca fue de 0,044 y 0,054 individuos/hora para los ríos y lagunas (o embalses), respectivamente. Se capturaron en total 17 individuos, $53 \%$ fueron adultos, $41 \%$ juveniles y $6 \%$ alevines. La relación longitud-peso encontrada indica que el coeficiente de crecimiento de la especie fue alométrico positivo. Se corroboró que $E$. mutisii se distribuye geográficamente en un rango altitudinal de 2540-3000 m s. n. m., aunque no se registró en las cuencas de los ríos Bogotá, Veraguas, Ubaté, Suárez y Frío. El declive de estas poblaciones se debe principalmente a factores antrópicos, como la transformación y fragmentación de los ecosistemas, la introducción de especies invasoras y la contaminación de los cuerpos hídricos.

Palabras claves: colecciones biológicas, pesca eléctrica, pez endémico, plan de conservación, relación peso-longitud

\section{Abstract}

Eremophilus mutisii (Humboldt, 1805), is an endemic fish to the Cundiboyacense plain, currently classified as vulnerable. The national programme to the catfish conservation, was used as a source for implementing the conservation plan in the CAR Cundinamarca in 2018. The present study performed the conservation status diagnosis of $E$. mutisii in Cundinamarca region which provides the basis for this conservation plan. In accordance, catfish geographical distribution was investigated by accessing biological collection information and conducting fish surveys of both lentic and lotic systems in pre-selection sites identified by the CAR officials and the research team. E. mutisii presence was evaluated in the Suárez, Bogotá (high basin), Ubaté, Veraguas, Frío, Aguas Claras and Muña rivers, also in the Fúquene lagoon and the Neusa and Tominé reservoirs. An electric fishing technique and physicochemical parameters measurement were used as the fish sampling approach of this study. The catfish catch per unit effort was 0.054 and 0.044 individuals/hour for rivers and

\footnotetext{
1. Centro de Estudios en Hidrobiología CEH, Universidad Manuela Beltrán, Bogotá, Colombia.

2. Dirección de Recursos Naturales de la Corporación Autónoma Regional de Cundinamarca CAR, Bogotá, Colombia.

* Autor de correspondencia: <ciromar.lemus@gmail.com>
} 
lagoons (or reservoirs), respectively. A total of 17 individuals were captured, $53 \%$ of them were adults, $41 \%$ juveniles and $6 \%$ small fry. The length-weight relationship found indicates that the species growth was positive allometric. It was confirmed that catfish is geographically distributed in an elevation range of 25403000 masl, although it was not reported in the Bogotá, Veraguas, Ubaté, Suarez and Frio rivers. The decline of $E$. mutisii populations is mainly due to anthropic factors, such as transformation and fragmentation of ecosystems, introduction of invasive species and water bodies contamination.

Keywords: biological collections, conservation plan, electric fishing, endemic fish, weight-length ratio

\section{INTRODUCCIÓN}

El pez capitán de la sabana (Eremophilus mutisii Humboldt, 1805) es una especie endémica y emblemática del altiplano cundiboyacense que se distribuye naturalmente en el rango altitudinal de los 2500 a 3100 m s. n. m. (Bastidas y Lemus, 2012; LemusPortillo et al, 2017; Maldonado-Ocampo, Vari, y Usma, 2008). Desde tiempos inmemoriales, este pez ha sido una fuente importante de alimento para los habitantes de esta región de la Cordillera Oriental de los Andes colombianos. Sin embargo, el actual deterioro de los recursos hídricos y la reducción del número de ejemplares han disminuido considerablemente su pesca, a excepción de sitios donde aún habita con cierta abundancia (Rodríguez-Forero, 2000).

La información disponible evidencia la reducción gradual del tamaño y densidad de las poblaciones de $E$. mutisii en ciertos ecosistemas lénticos, como las lagunas de Fúquene y Suesca (Mojica, Usma, ÁlvarezLeón, y Lasso, 2012), junto con la extinción local en varios ecosistemas lóticos del páramo de Cruz Verde o la cuenca media del río Bogotá (DoNascimiento, Prada-Pedreros, y Guerrero-Kommritz, 2014; PradaPedreros, Rivera-Rondón, y Guerrero- Kommritz, 2006). Las principales amenazas a la especie se encuentran en la degradación y contaminación de los ecosistemas acuáticos por la gran presión demográfica que ejerce el $19 \%$ de la población total del país asentada en la gran urbe de Bogotá y 17 municipios aledaños (Corporación Autónoma Regional de Cundinamarca, 2009c). Todo esto indica que el estado de conservación de E. mutisii ha empeorado si se considera el aumento de su categoría de amenaza, de casi amenazada (NT) a vulnerable (VU), según las dos últimas evaluaciones del libro rojo de peces dulceacuícolas de Colombia realizadas en un período de diez años (Mojica et al, 2012).
Por tal motivo, se elaboró el programa nacional para la conservación de E. mutisii (Lemus-Portillo et al, 2017), el cual diseña las acciones prioritarias a implementar con una visión a 15 años, a fin de revertir el riesgo de extinción que enfrenta la especie. Igualmente, este programa estableció la participación conjunta entre instituciones y actores sociales del país para el avance del proceso de conservación, cuyo primer aporte fue el desarrollo del plan de conservación y manejo del pez capitán de la sabana que la Corporación Autónoma Regional de Cundinamarca (CAR) implementó en el departamento de Cundinamarca (Rojas, Echavarria, y Lemus-Portillo, 2018). Los objetivos principales de este plan fueron el diagnóstico del estado ecológico de la especie y la verificación de la distribución real del pez, dentro del territorio de la jurisdicción CAR Cundinamarca, en diferentes ecosistemas lóticos y lénticos previamente identificados; permitiendo la implementación del plan de conservación y manejo necesario para la toma de decisiones por parte de las autoridades ambientales, mediante la alianza con instituciones científicas, educativas, gubernamentales, privadas y comunidades locales. Este estudio presenta los resultados de la evaluación del estado de conservación y distribución geográfica de las poblaciones de E. mutisii en la jurisdicción CAR Cundinamarca, basado tanto en el número de individuos capturados como en la revisión de la distribución natural de la especie a partir de colecciones biológicas.

\section{MATERIALES Y MÉTODOS}

\section{Descripción de la especie}

El pez capitán de la sabana (figura 1) es la especie más grande de la familia Trichomycteridae (Dahl, 1971), perteneciente al orden Siluriformes, que comúnmente se conocen como "peces gato o bagres". 
Las características morfológicas más sobresalientes de E. mutisii son: a) cuerpo cilíndrico de forma serpentiforme, desprovisto de escamas; cabeza aplanada a nivel dorso-ventral (Mayorga, 1992), con presencia de tres pares de barbillones táctiles (narinal, maxilar y rictal) típico de los bagres y dos pares de narinas separadas entre sí (Mojica et al, 2012); b) ojos pequeños en comparación con la proporción cefálica, de aproximadamente 1,2-2,6 $\mathrm{mm}$ de diámetro en proporción con la talla del individuo (Lemus-Portillo et al, 2017); c) cinco aletas radiadas (una dorsal, dos pectorales, una anal y una caudal de aspecto redondeada) sin aletas pélvicas, carácter típico de la especie a nivel de género; d) variabilidad cromática corporal, ya sea de color verde oliva, azul o negro, siempre acompañado de manchas amarillas o blancas de aspecto vermicular (Bastidas y Lemus, 2014, Lemus-Portillo et al, 2017); aunque se pueden encontrar ejemplares albinos denominados "capitán rey" (Mojica et al, 2012) que varían entre la forma completamente rosada hasta la casi vermiculada con algunas manchas incoloras (Miles, 1942; Mojica, Castellanos, Usma, y Álvarez-León, 2002); e) boca amplia en posición subterminal (Rosado y González, 2007); y f) opérculos que presentan pequeñas espinas que emplean para enterrarse o sujetarse entre la vegetación sumergida o emergente (Bastidas y Lemus, 2014).

Con respecto a la biometría de E. mutisii, se reportan tallas de longitud total hasta $30 \mathrm{~cm}$, pero según Dahl
(1971) pueden alcanzar los $50 \mathrm{~cm}$; siendo las hembras de mayor talla que los machos (Bastidas y Lemus, 2014). De acuerdo con registros consultados de colecciones biológicas y reportes hechos en este estudio, las tallas (longitud total) del pez capitán de la sabana para el área de influencia de la jurisdicción CAR son las siguientes: 1) ríos, $24 \mathrm{~cm}$ para machos y $28 \mathrm{~cm}$ para hembras; 2) lagunas, $20 \mathrm{~cm}$ para machos y $24 \mathrm{~cm}$ para hembras; y 3) embalses, $22 \mathrm{~cm}$ para machos y 25 $\mathrm{cm}$ para hembras. En referencia al peso, las hembras registran, en promedio, $230 \mathrm{~g}$ y los machos $120 \mathrm{~g}$, condición que se hace más notoria durante el período de ovulación de las hembras cuando su vientre es más prominente.

\section{Área de estudio}

La distribución de E. mutisii en Cundinamarca se evaluó a partir de la recopilación de registros de colecciones biológicas y salidas de campo a sitios previamente identificados entre la CAR y el grupo investigador para confirmar la presencia de la especie o llenar vacíos de información. Para la selección de los sitios de muestreo se tuvieron en cuenta el conocimiento de los especialistas y las condiciones ambientales, como el rango de temperatura del agua entre 9 y $20^{\circ} \mathrm{C}$, el rango altitudinal que se enmarcó entre 2400 y $3100 \mathrm{~m}$ s. n. m., las concentraciones de oxígeno disuelto por encima de $2 \mathrm{mg} / \mathrm{L}$ y la existencia de distintas unidades hidromorfológicas (Bastidas y Lemus, 2012).

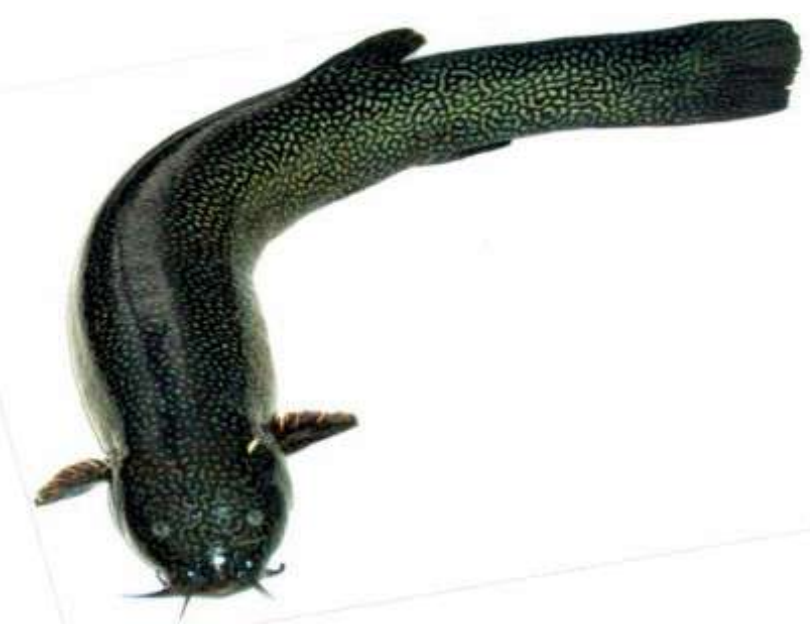

Figura 1. Ejemplar de Eremophilus mutisii. Foto: Ciromar Lemus-Portillo 
Con la información obtenida se realizaron salidas de campo entre junio y octubre de 2018, en época seca y húmeda, para comprobar la presencia de la especie en los ríos Suárez, Bogotá (cuenca alta), Ubaté, Veraguas, Frío, Aguas Claras y Muña; la laguna de Fúquene y los embalses del Neusa y Tominé (figura 2). En cada salida se estableció una zona de muestreo que permitiera contener la máxima heterogeneidad ecológica del cuerpo de agua representada por la cobertura de macrófitas, la forma de la orilla y el tipo de hábitats, mesohábitats o unidades hidromorfológicas (UHMs) (Bastidas y Lemus, 2012; Parasiewicz, Gortazar, Sánchez, y García de Jalón, 2009). Estas condiciones ambientales favorecen la existencia de hábitats para las especies ícticas bajo la sombrilla de las macrófitas (Jacobsen y Dangles,
2017; Smokorowski y Pratt, 2007). Igualmente, se eludieron los vertimientos puntuales o cualquier tipo de represamiento que pudieran afectar la distribución natural de las especies ícticas, de manera que solo fueron incluidos hábitats adecuados para ellas (Pulido-Méndez, 2013).

\section{Muestreo}

En cada ecosistema acuático se establecieron tres estaciones de muestreo en las que se realizó el mismo esfuerzo de captura. Igualmente, en cada estación se dispuso una longitud mínima de muestreo en función del ancho del río en estudio (tabla 1), mientras que en lagunas y embalses se instalaron tres áreas de muestreo cada una de 20 x 10 m. En estos ecosistemas

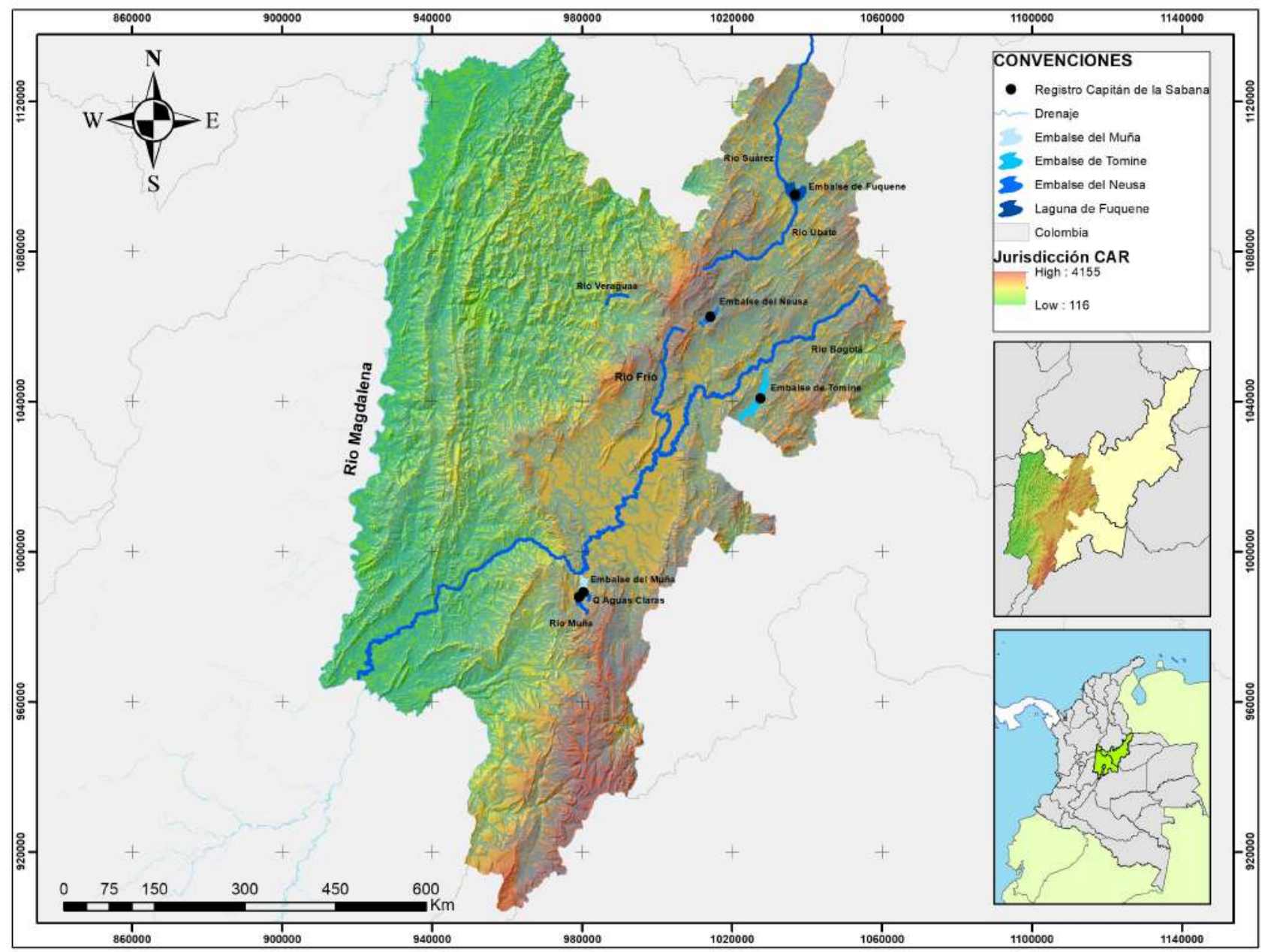

Figura 2. Distribución geográfica de Eremophilus mutisii en la jurisdicción CAR-Cundinamarca. 
Tabla 1. Criterios para el establecimiento de áreas de muestreo en ecosistemas lóticos.

\begin{tabular}{l|l|l|l}
\hline Ancho del río $(\mathbf{m})$ & Longitud mínima de muestreo $(\mathbf{m})$ & Ancho mínimo de muestreo & Abundancia \\
\hline$<5$ & 20 & Completa & Absoluta \\
\hline $5-15$ & 50 & Completa & Absoluta \\
\hline$>15$ & $>50$ & Margen fluvial & Relativa \\
\hline
\end{tabular}

lénticos, los muestreos siempre se realizaron en lugares que albergan condiciones naturales del cuerpo de agua, como la presencia de macrófitas flotantes o emergentes que permitieran la existencia de sitios de refugio para la especie.

La captura de peces se realizó mediante la técnica de pesca eléctrica, que es muy utilizada en ríos y agua estancada vadeable pues resulta inocua para los peces que se recuperan sin efectos secundarios tras la descarga eléctrica (Sostoa, García de Jalón, y García, 2005). Sin embargo, los procedimientos de pesca dependen de ciertas características físicas, como la profundidad del agua, el tamaño de la masa de agua, la velocidad, la conductividad eléctrica y el tamaño de la especie a capturar (Elosegi y Sabater, 2009; Lobón-Cerviá, 1991). Por lo tanto, una vez se registró la medida de conductividad, inmediatamente se calibró la frecuencia y la longitud de la descarga eléctrica generada por el equipo portátil marca SAMUS 1000. Este equipo emite una corriente continua pulsátil (CCP) de 300 a $600 \mathrm{~V}$ con $1,5 \mathrm{~A}$, que no resulta lesiva para los peces y es más segura para los investigadores.

Una vez aplicada la descarga eléctrica, dos personas se situaron con nasas de recolecta (abertura de $50 \mathrm{~cm}$, ojo de malla de $4 \mathrm{~mm}$, longitud de $100 \mathrm{~cm}$, diámetro en mango de aluminio de $3,2 \mathrm{~mm}$ ), detrás del portador de la red eléctrica recolectando aquellos peces que aturdidos por la electricidad fueron arrastrados por la corriente (Espinosa-Pérez, 2014). Además, la eficiencia de pesca incrementó al delimitar un área de muestreo aguas abajo del sitio de la descarga con una red de trasmallo, constituida por tres paños superpuestos y reforzados con anclajes que permitieron mantener estable su posición. El trasmallo retiene los individuos que quedan en estado de letargo y adormecimiento y que no logran ser recolectados por las nasas de captura superficial (Rojas et al, 2018).
La unidad de esfuerzo de muestreo se estableció mediante el método de pasadas sucesivas sin devolución, consistente en tres pasadas sobre un tramo definido del río. Cada pasada conlleva el mismo esfuerzo de pesca, esto es con idéntico número de personas e igual duración (30 min) por cada sitio de muestreo o mesohábitat. Así, el esfuerzo total de captura fue de una hora y treinta minutos para cada uno de los ríos evaluados. Los peces recolectados se depositaron en baldes con agua y una solución tranquilizante de forma que se facilitó su manipulación durante el registro morfológico y biométrico (Ginés, 2011), para luego ser liberados cuidadosamente en el cuerpo hídrico.

Por otra parte, para los muestreos en cuerpo lénticos no vadeables, como embalses y lagunas, la pesca se realizó de manera artesanal empleando una red de arrastre lineal de $40 \mathrm{~m}$ de longitud y un diámetro de malla de $3 \mathrm{~cm}$, con distribución de flotadores y pesas de 3,0 g a lo largo de la red. Al inicio del muestreo, la red se dispuso en el área de recolecta desde una embarcación, dejándola expuesta durante 4 h. Finalmente, se recogió la red mediante movimientos suaves de arrastre.

\section{Medidas biométricas}

A cada ejemplar capturado se le registró el peso (g), longitud furcal ( $\mathrm{mm})$, longitud total $(\mathrm{mm})$, ancho del cuerpo $(\mathrm{mm})$, ancho de la cabeza $(\mathrm{mm})$, conteo de aletas, características sexuales, estado de madurez del ejemplar (adulto, juvenil y alevín) y se le realizó el respectivo registro fotográfico. Se utilizaron las capturas realizadas, sin discriminar entre macho y hembras, para estimar la relación peso totallongitud total (PTLT) (Ricker, 1975), utilizando el modelo potencial o ecuación de alometría de Huxley y Teisser (1936): PT $=\boldsymbol{a} *(\mathbf{L T})^{b}$, donde $\mathbf{P T}$ es el peso total, LT es la longitud total, $\boldsymbol{a}$ es el intercepto y $\boldsymbol{b}$ es la pendiente de la curva. 


\section{RESULTADOS}

\section{Distribución geográfica}

La distribución geográfica de E. mutisii en Cundinamarca transcurre en un rango altitudinal de 25403000 m s. n. m., con un coeficiente de variación del 5,3\%, según los registros históricos consultados (tabla 2). Estos registros refieren localidades, como los embalses de Muña, Sisga y Tominé; las lagunas de Fúquene y Suesca; y los ríos Bogotá, Checua, Frío, Teusacá y Ubaté. Específicamente, se encontraron registros de la cuenca media del río Bogotá en un rango altitudinal entre 2540 y $2625 \mathrm{~m} \mathrm{s.} \mathrm{n.} \mathrm{m.} \mathrm{(CV} \mathrm{=}$ $0,86 \%$ ) que corresponden a capturas antiguas hechas en inmediaciones de la ciudad de Bogotá o más recientes en alrededores del municipio de Suesca. Los registros a mayor altura (2850-3000 m s. n. m.) se localizan en el embalse de Neusa, la laguna de Suesca y el río Teusacá. El mayor número de registros se establecieron entre los 2540 y 2730 m s. n. m., en sitios representativos como la laguna de Fúquene, los embalses del Muña, Sisga y Tominé, y los ríos Bogotá, Checua, Frío y Ubaté.

Tabla 2. Registros históricos de localidades y poblaciones de Eremophilus mutisii en Cundinamarca.

\begin{tabular}{|c|c|c|c|c|}
\hline Localidad & Año & Colectores & Poblaciones (n) & Altitud (m s. n. m.) \\
\hline Embalse Muña & 1982 & Cala, P. & 2570 & 2570 \\
\hline \multirow{3}{*}{ Embalse Neusa } & 1992 & Beltrán & 150 & 2970 \\
\hline & 1993 & Pinilla et al. & 96 & 2975 \\
\hline & 2010 & Operarios CAR & 210 & 2970 \\
\hline Embalse Sisga & 1996 & Colección PUJ & 3 & 2660 \\
\hline \multirow{2}{*}{ Embalse Tominé } & 1982 & Flórez y Sarmiento & 177 & 2580 \\
\hline & 2015 & Echavarria y Lemus & 29 & 2585 \\
\hline \multirow{7}{*}{ Laguna Fúquene } & 1971 & Páez, C. & 5 & 2540 \\
\hline & 1992 & Beltrán & 644 & 2540 \\
\hline & 1992 & Mayorga & 68 & 2540 \\
\hline & 2005 & Valderrama-Hernández & 620 & 2540 \\
\hline & 2006 & Ortega-Lara et al. & 2 & 2545 \\
\hline & 2009 & Anderson & 1878 & 2540 \\
\hline & 2015 & Echavarria y Lemus & 16 & 2540 \\
\hline Laguna Hernández* & 1958 & Medem, F. y Jaramillo, R. & 8 & - \\
\hline Laguna Suesca & 1994 & Piedrahita-Ruiz & 52 & 2850 \\
\hline Lagunas Sabana de Bogotá** & 1993 & Hernández, G. & 2 & 2550 \\
\hline \multirow{11}{*}{ Río Bogotá } & 1943 & Miles et al. & 225 & 2549 \\
\hline & 1958 & Medem, F. & 5 & 2550 \\
\hline & 1960 & Toro, G. & 1 & 2550 \\
\hline & 1966 & Huertas, G. & 14 & cf. 2540 \\
\hline & 1971 & Dahl & 460 & 2550 \\
\hline & 2004 & Vásquez et al. & 12 & 2570 \\
\hline & 2004 & Colección PUJ & 4 & 2570 \\
\hline & 2005 & Jiménez y Pinto & 50 & 2560 \\
\hline & 2006 & Rodríguez, A. et al. & 70 & 2625 \\
\hline & 2006 & Alvarez, R. y Prada-Pedreros, S. & 1 & 2570 \\
\hline & 2007 & Rodríguez, A. et al. & 61 & 2570 \\
\hline Río Checua & 2013 & Bastidas y Lemus & 13 & 2570 \\
\hline \multirow{2}{*}{ Río Frío } & 2012 & Bastidas y Lemus & 71 & 2565 \\
\hline & 2015 & Lemus y Echavarria & 37 & 2565 \\
\hline Río Teusacá & 1982 & Flórez y Sarmiento & 4 & 3000 \\
\hline \multirow{2}{*}{ Río Ubaté } & 2006 & Alvarez, R. et al. & 1 & 2543 \\
\hline & 2016 & Lemus y Ovalle & 13 & 2730 \\
\hline
\end{tabular}

*La localidad Laguna Hernández debe confirmarse.

** La descripción indica que la Laguna de Sabana de Bogotá está canalizada por parcelación, con vallados del Río Subachoque (cf.); cf. Confirmar. 
En los muestreos de campo, la presencia de $E$. mutisii se registró en la Laguna de Fúquene, los embalses de Neusa y Tominé y los ríos Aguas Claras y Muña. Mientras que estuvo ausente en los ríos Veraguas, Ubaté, Bogotá, Suárez y Frío. La ausencia del capitán de la sabana demuestra cambios en las condiciones ambientales de estos ríos, que incluyen el deterioro de la calidad del agua, las modificaciones hidromorfológicas por dragado y la canalización de cauces, además de la introducción de especies invasoras. Sin duda, las perturbaciones antrópicas observadas han afectado la supervivencia de las poblaciones y restringido la distribución natural del pez capitán de la sabana en todas las cuencas de la jurisdicción CAR Cundinamarca.

De acuerdo con los parámetros fisicoquímicos medidos in situ (tabla 3) se puede constatar que 4 de los 5 ríos en los que no se verificó la presencia de E. mutisii se encuentran en un rango altitudinal óptimo (24003100 m s. n. m.) para la especie, a excepción del río Veraguas (1570 m s. n. m.); también cumplen en su totalidad con las condiciones fisicoquímicas del agua para la presencia del capitán de la sabana a saber: temperatura entre 9 y $20{ }^{\circ} \mathrm{C}$, y concentración de oxígeno disuelto mayor a $2 \mathrm{mg} / \mathrm{L}$ (Bastidas y Lemus, 2014), a excepción del río Suárez. En efecto, los cuer- pos hídricos que incumplen con estos condicionantes son el río Veraguas, que se encuentra por debajo de la altitud óptima encontrada para el pez capitán, y el río Suárez, que posee condiciones críticas de oxígeno con un rango entre valores cercanos a 0 y 2,75 mg/L, que determinan condiciones notorias de anoxia e hipoxia (Roldán y Ramírez, 2008).

Un caso particular lo constituye el río Veraguas, afluente del río Negro localizado al noroccidente de Cundinamarca, el cual fue monitoreado para corroborar la información registrada de la presencia del pez capitán de la sabana por la CAR Cundinamarca; ante lo cual se confirma que la especie no se halla en altitudes por debajo de los $2400 \mathrm{~m}$ s. n. m. De igual forma, los altos niveles de sólidos suspendidos totales, que presenta el río Veraguas y toda la cuenca del río Negro (1723 mg/L) (CAR, 2009a), pueden tener efectos transgeneracionales sobre las poblaciones del pez capitán, que se traducen en cambios comportamentales y fisiológicos que en corto y largo plazo reflejan limitaciones en migraciones, desoves o movimientos de las poblaciones (Kjelland, Woodley, Swannack, y Smith, 2015).

En todos los sistemas acuáticos estudiados en Cundinamarca se capturaron 17 ejemplares, 13 de los

Tabla 3. Parámetros fisicoquímicos medidos in situ en los sitios de muestreo.

\begin{tabular}{|c|c|c|c|c|c|c|}
\hline Localidad & Altitud (m s. n. m.) & Temperatura $\left({ }^{\circ} \mathrm{C}\right)$ & $\mathrm{pH}$ & $O D(m g / L)$ & $\begin{array}{l}\text { Conductividad } \\
(\mu \mathrm{S} / \mathrm{cm})\end{array}$ & $\begin{array}{l}\text { Turbiedad } \\
\text { (NTU) }\end{array}$ \\
\hline \multirow{3}{*}{ Río Veraguas } & 1570 & 18,5 & 8,19 & 7,98 & 218,0 & 21,54 \\
\hline & 1570 & 8,4 & 8,19 & 7,96 & 216,0 & 24,85 \\
\hline & 1570 & 18,5 & 8,26 & 7,85 & 218,0 & 19,16 \\
\hline \multirow{5}{*}{ Río Ubaté } & 2615 & 13,6 & 7,22 & 7,77 & 87,1 & 10,45 \\
\hline & 2615 & 13,6 & 7,65 & 7,77 & 86,6 & 10,69 \\
\hline & 2560 & 13,9 & 7,57 & 7,59 & 99,6 & 10,79 \\
\hline & 2554 & 12,9 & 7,4 & 7,12 & 106,2 & 12,83 \\
\hline & 2559 & 14,1 & 6,96 & 6,41 & 163,6 & 51,00 \\
\hline \multirow{2}{*}{ Río Suárez } & 2548 & 18,8 & 6,61 & 1,51 & 206,0 & 16,21 \\
\hline & 2554 & 16,6 & 6,43 & 0,02 & 223,0 & 36,15 \\
\hline \multirow{2}{*}{ Río Bogotá } & 2733 & 12,7 & 6,80 & 7,80 & 28,6 & 18,64 \\
\hline & 2625 & 13,9 & 6,74 & 5,61 & 60,4 & 116,00 \\
\hline Río Frío & 2560 & 14,1 & 7,07 & 6,93 & 67,3 & 29,26 \\
\hline Aguas Claras & 2584 & 14,1 & 6,49 & 7,78 & 30,1 & 4,85 \\
\hline Río Muña & 2632 & 13,4 & 7,18 & 7,40 & 69,6 & 13,22 \\
\hline Fúquene & 2562 & 16,6 & 7,00 & 3,40 & 205,0 & 9,75 \\
\hline Neusa & 2985 & 14,5 & 6,46 & 4,20 & 64,8 & 25,24 \\
\hline Tominé & 2590 & 18,0 & 8,00 & 7,7 & 38,8 & 37,11 \\
\hline
\end{tabular}


cuales se recolectaron en sistemas lénticos y 4 en los sistemas lóticos, para un esfuerzo de captura de 0,054 y 0,044 individuos/hora en cada uno de estos sistemas, respectivamente. La proporción observada de sexos de estos ejemplares fue 1:1, con una mayoría de adultos $(55 \%)$ y juveniles (41\%), y una baja proporción de alevines (6\%) (figura 3). En relación con las tallas y pesos, los individuos adultos capturados en la laguna Fúquene se encontraron en una talla media de $20,6 \pm 1,56 \mathrm{~cm}$ y un peso medio de $87,6 \pm 17,32 \mathrm{~g}$ (tabla 4). Sin embargo, se capturaron dos individuos adultos, un macho en el embalse Neusa y una hembra en el río Aguas Claras, ambos de $31 \mathrm{~cm}$ de talla y un peso de 419 g para el macho y de 480 g para la hembra. Por otro lado, los ejemplares juveniles capturados en la laguna Fúquene presentaron una talla media de $16,57 \pm 0,65 \mathrm{~cm}$ y un peso medio de $52 \pm 7,30 \mathrm{~g}$, mientras aquellos capturados en los ríos Aguas Claras y Muña presentaron tallas y pesos menores de $7,46 \pm 2,96 \mathrm{~cm}$ y $5,66 \pm 5,51 \mathrm{~g}$, respectivamente.

A partir de las medidas de longitud total y peso, se estimó la relación peso total-longitud total para los 17 ejemplares capturados, cuyo resultado fue la ecuación: $\mathrm{PT}=(0,00057) *(\mathrm{LT})^{3,17}$, la cual indica que el coeficiente de crecimiento de la especie fue de tipo alométrico positivo $(\mathrm{n}=17 ; b>3,0 ; p<0,050)$ (figura 4).

\section{DISCUSIÓN}

El pez capitán de la sabana es una especie endémica con distribución geográfica en el altiplano cundiboyacense, de importancia en la región debido a que hace parte del sustento alimenticio de los pobladores (Lemus-Portillo et al, 2017). Por esta razón, se evaluó su presencia en los ecosistemas acuáticos del área de jurisdicción de la CAR Cundinamarca, destacándose que en las últimas décadas su presencia se ha visto amenazada, al punto que la especie se encuentra en condición vulnerable (VU); este declive obedece a factores antrópicos, fragmentación y contaminación de su hábitat, así como la presencia de especies invasoras en su territorio (Bastidas y Lemus, 2014).

La especie ocupa un rango altitudinal de 2540-3000 m s. n. m., con un coeficiente de variación del 5,3\%, según los registros históricos consultados. Aunque no se haya registrado la presencia del capitán de la sabana en los cinco sistemas lóticos referenciados anteriormente, por ejemplo, en el río Suárez fueron recolectados varios especímenes del cangrejo rojo americano o langostilla (Procambarus clarkii) y en

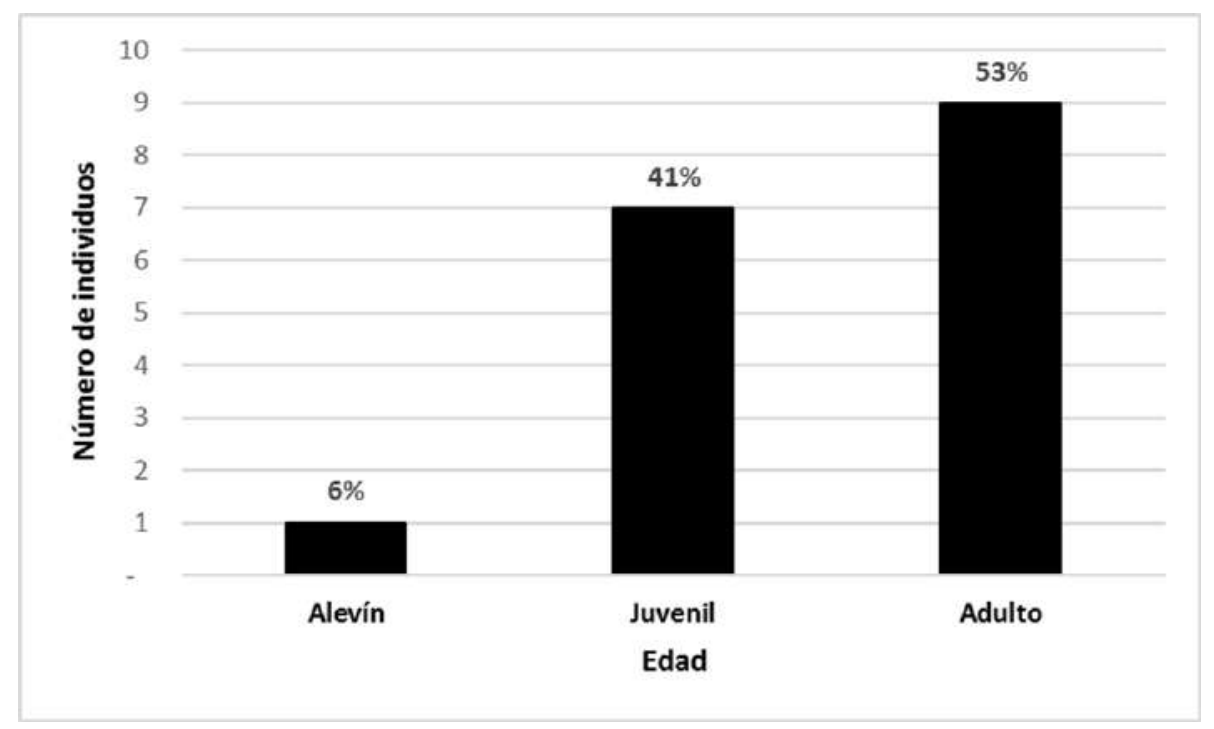

Figura 3. Estructura de edades de los individuos de Eremophilus mutisii capturados (LT) ${ }^{3,17}$, la cual indica que el coeficiente de crecimiento de la especie fue de tipo alométrico positivo $(n=17 ; b>3,0 ; p<0,050)$ (figura 4). 


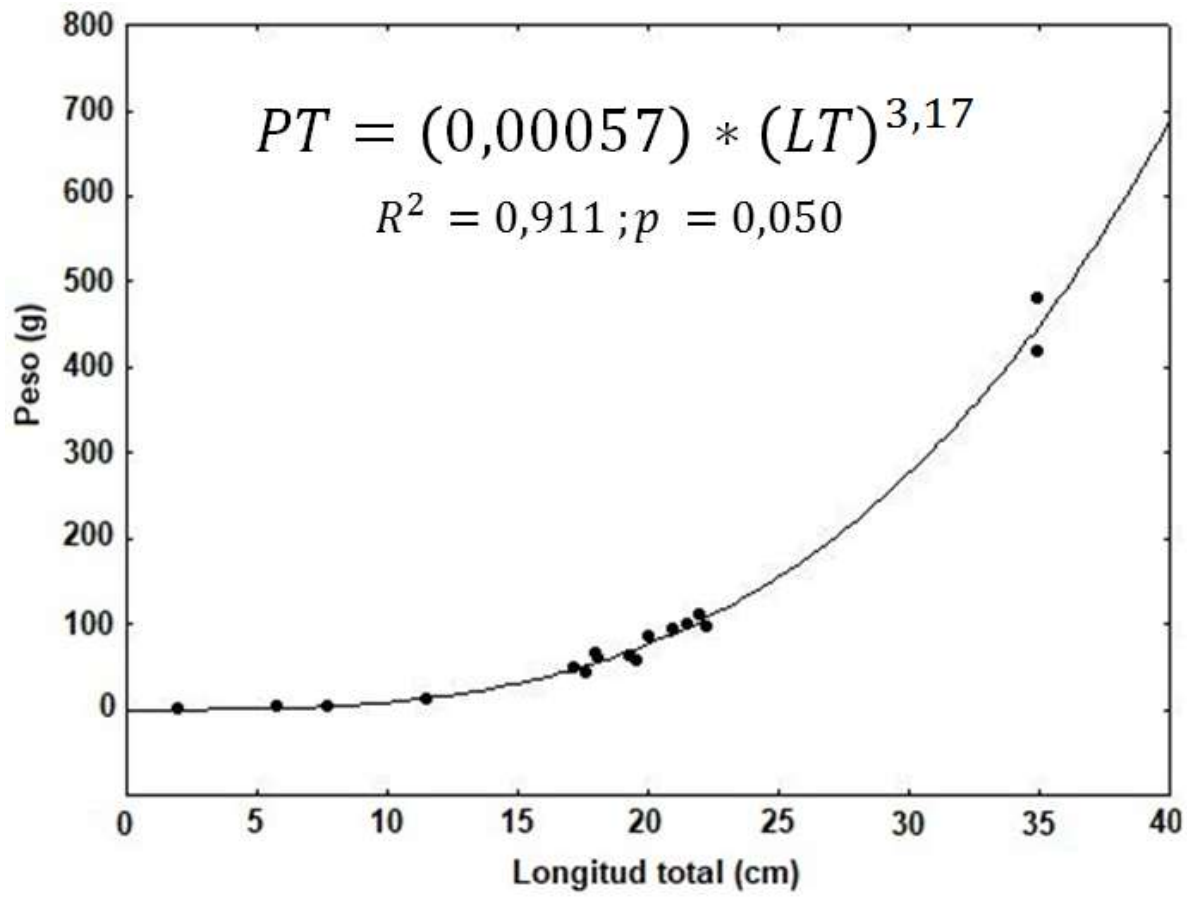

Figura 4. Ecuación de alometría para Eremophilus mutisii en Cundinamarca

Tabla 4. Medidas de longitud, peso, sexo y edad de los individuos capturados de Eremophilus mutisii.

\begin{tabular}{|c|c|c|c|c|c|c|c|c|}
\hline Localidad & $\begin{array}{l}\text { Longitud } \\
\text { furcal }(\mathrm{cm})\end{array}$ & $\begin{array}{l}\text { Longitud } \\
\text { total }(\mathrm{cm})\end{array}$ & $\begin{array}{l}\text { Ancho cuer- } \\
\text { po }(\mathrm{cm})\end{array}$ & $\begin{array}{l}\text { Ancho cabe- } \\
\mathrm{za}(\mathrm{cm})\end{array}$ & $\begin{array}{l}\text { Altura cuer- } \\
\text { po }(\mathrm{cm})\end{array}$ & Peso (g) & Sexo & Edad \\
\hline \multirow{11}{*}{ Laguna Fúquene } & 16,0 & 18,0 & 2,5 & 2,5 & 3,0 & 66,00 & $\mathrm{H}$ & Adulto \\
\hline & 18,0 & 20,0 & 3,2 & 2,6 & 3,8 & 86,00 & $\mathrm{H}$ & Adulto \\
\hline & 20,0 & 22,0 & 3,2 & 2,8 & 3,5 & 110,00 & H & Adulto \\
\hline & 19,5 & 21,5 & 3,0 & 3,0 & 3,5 & 98,00 & $\mathrm{H}$ & Adulto \\
\hline & 20,3 & 22,3 & 3,0 & 1,8 & 4,0 & 97,00 & $\mathrm{H}$ & Adulto \\
\hline & 20,0 & 21,0 & 3,0 & 2,9 & 3,0 & 93,00 & M & Adulto \\
\hline & 17,4 & 19,3 & 2,8 & 2,5 & 3,0 & 63,00 & $M$ & Adulto \\
\hline & 17,5 & 19,6 & 2,8 & 2,5 & 2,8 & 56,00 & $\mathrm{H}$ & Juvenil \\
\hline & 16,0 & 17,6 & 2,5 & 2,0 & 3,0 & 44,00 & H & Juvenil \\
\hline & 16,5 & 18,1 & 2,8 & 2,4 & 3,0 & 60,00 & $\mathrm{H}$ & Juvenil \\
\hline & 16,3 & 17,2 & 2,8 & 2,5 & 2,7 & 48,00 & $M$ & Juvenil \\
\hline Embalse Neusa & 31,0 & 35,0 & 5,0 & 3,7 & 5,0 & 419,00 & $M$ & Adulto \\
\hline Embalse Tominé & 1,8 & 2,0 & 0,2 & 0,3 & 0,3 & 0,07 & Ind & Alevín \\
\hline \multirow{3}{*}{ Aguas Claras } & 31,0 & 35,0 & 4,5 & 3,5 & 5,9 & 480,00 & $\mathrm{H}$ & Adulto \\
\hline & 6,8 & 7,7 & 1,0 & 1,2 & 1,3 & 3,00 & $M$ & Juvenil \\
\hline & 4,9 & 5,8 & 0,8 & 0,9 & 0,7 & 2,00 & $M$ & Juvenil \\
\hline Río Muña & 10,7 & 11,5 & 1,34 & 1,39 & 1,5 & 12,00 & $M$ & Juvenil \\
\hline
\end{tabular}


los ríos Bogotá, Ubaté y Frío otros tantos de trucha arcoíris (Oncorhynchus mykiss), ambas especies consideradas invasoras (Baptiste et al, 2010). En contraste, en todos los sistemas lénticos muestreados se estableció la presencia de E. mutisii, siendo la laguna de Fúquene el sistema acuático con la mayor capacidad de soportar y mantener una población estable del pez capitán, aunque sea notoria también la presencia de varias especies introducidas, como la carpa (Cyprinus carpio) y la langostilla (P. clarki). Por último, debe considerarse que estos ecosistemas lénticos cumplen con condiciones de hábitat (temperatura, altitud y concentración de oxígeno disuelto), así como de presencia de relictos de vegetación sumergida, flotante y emergente, que brindan hábitats adecuados para la presencia de E. mutisii (Bastidas y Lemus, 2014).

En conjunto, la abundancia de macrófitas y de otros factores, conlleva a una baja calidad del agua dada por la alta carga orgánica y de otros contaminantes que se dispersan o concentran dependiendo del flujo y el área del ecosistema acuático; situación que es común a todos los cuerpos lóticos evaluados en este estudio (Lemus-Portillo et al, 2017). Por lo tanto, un manejo adecuado consiste en reducir la concentración de las cargas contaminantes que alcanzan los cuerpos hídricos, mediante el control de los vertimientos en la fuente, siendo esta la causa principal del desplazamiento del pez capitán en áreas donde anteriormente fue registrado (Bastidas y Lemus, 2014). No obstante, la presencia de E. mutisii en ecosistemas lénticos que hoy en día se encuentran altamente contaminados y alterados, como el caso de la laguna de Fúquene y en menor grado los embalses de Neusa y Tominé, puede deberse a la ecología de estos sistemas lénticos que les permiten auto depurarse o deshacerse de los contaminantes por diferentes procesos químicos y físicos (Roldán y Ramírez, 2008). En el caso de la laguna de Fúquene, el volumen de agua aún es alto y la dinámica hídrica favorecida por la entrada del río Ubaté y el único desagüe natural por el río Suárez, facilitan la depuración natural de la laguna.

En el área de estudio es muy frecuente la existencia de especies introducidas, como el cangrejo rojo americano o langostilla ( $P$. clarki), carpa $(C$. carpio) y trucha arcoíris (O. mykiss) en la laguna de Fúquene y los embalses del Neusa y Tominé, especies invasoras que se convierten en una amenaza real y potencial para la supervivencia de las poblaciones de E. mutisii y otras especies de peces altoandinos (guapucha, Grundulus bogotensis; y capitanejo, Trichomycterus bogotense), ya que se presume la depredación de las ovas y alevines de estos peces nativos por O. mykiss (Bastidas y Lemus, 2014; Elvira y Almodóvar, 2010).

En efecto, $P$. clarki es considerada una especie invasiva debido a su capacidad de desestabilizar los ecosistemas al momento de ser introducida, gracias a los cambios en sus hábitos alimenticios (CAR 2016b; Pachón y Valderrama, 2018). Esto implica un impacto en todos los niveles de la cadena trófica, puesto que en épocas de lluvia esta especie es omnívora, detritívora y filtradora, pero en época seca se convierte en herbívora (Correia, 2002; Correia, Bandeira, y Anastácio, 2007). Se ha comprobado que en la laguna de Fúquene, la langostilla depreda peces como el pez capitán de la sabana (Arias-Pineda y Pedroza-Martínez, 2018; Pachón y Valderrama, 2018). Entretanto, la carpa es considerada como una de las 100 especies invasoras más dañinas a nivel ecosistémico del país (Galván-Guevara y De la Ossa, 2011; Gutiérrez-Bonilla y Álvarez-León, 2011) puesto que al ser bentívora, genera movimientos de sedimentos en la columna de agua, alterando así su calidad debido al aumento de la turbiedad y las concentraciones de nitrógeno y fósforo, que por consiguiente acrecienta la proliferación de macrófitas flotantes (García-Berthou et al, 2015).

Otra amenaza detectada fue la sobrepesca, la cual se involucra significativamente en el declive del pez capitán. Si bien existe una restricción de la pesca de E. mutisii dada la categoría de vulnerabilidad en que se encuentra, debiendo ser acatada por los pescadores durante sus faenas de pesca para garantizar su preservación (Caicedo, 2017; Lemus-Portillo et al, 2017; Narváez-Barandica, Herrera-Pertuz, y BlancoRacedo, 2008). En síntesis, las actividades antrópicas que impactan directamente sobre los cuerpos lóticos han afectado negativamente la presencia de $E$. mutisii. Pese a esto, las poblaciones más estables de la especie se encuentran en cuerpos lénticos, sin importar las continuas amenazas que enfrentan en general todos estos ecosistemas en el departamento 
de Cundinamarca, como la sobrepesca, la pérdida de áreas originales, la descarga de vertimientos y la introducción de especies invasoras.

El dragado y las obras de conformación de cauces, con la intención de profundizar los ríos y así prevenir inundaciones en época de caudal máximo, se realizan de forma cotidiana por parte de la autoridad ambiental en cumplimiento de la Ordenanza 210/2011, por la cual la Asamblea Departamental de Cundinamarca "adopta directivas para la protección de los cauces y eliminación de riesgos por inundación en el territorio del Departamento de Cundinamarca". Sin embargo, este tipo de actividades implican variaciones a nivel fisicoquímico, biológico y ecosistémico, relacionadas directamente con la destrucción del hábitat del pez capitán (Bastidas y Lemus, 2014; Lemus-Portillo et al, 2017).

Basado en lo anterior, se infiere que cada una de las actividades antrópicas realizadas generan impactos sobre los ecosistemas lóticos y lénticos, que conducen al deterioro de la calidad del agua y la destrucción del hábitat del pez capitán. Esto ocasiona la disminución del tamaño poblacional en áreas altamente perturbadas del ecosistema acuático, lo que consecuentemente afecta los patrones naturales de movimiento, restringiendo la especie a áreas de distribución más pequeñas y aisladas entre sí.

En este estudio no se realizaron análisis fisicoquími$\cos$ ex situ que permitieran conocer el tipo de carga contaminante en los ríos. Aunque, según los objetivos de calidad del agua trazados por la CAR (2009b), se sugiere que la supervivencia de las poblaciones de peces está relacionada con el deterioro progresivo de la calidad de agua de los ríos Ubaté, Suárez cuenca alta del río Bogotá y Frío a medida que reciben cargas contaminantes puntuales y difusas (CAR, 2016a). Las características de hábitat detectadas en los diferentes cuerpos lóticos y lénticos estudiados, permiten suponer que la distribución restringida del pez capitán de la sabana se debe a la pérdida de unidades hidromorfológicas y de conectividad entre estas (Vezza, Parasiewicz, Spairani, y Comoglio, 2014), la contaminación de las fuentes hídricas y la introducción de especies exóticas, como la trucha arcoíris $(O$. mykiss) cuya alta abundancia, especialmente en la cuenca alta del río Bogotá, es un factor biológico que puede explicar el detrimento poblacional del pez capitán (Lemus-Portillo et al, 2017; Rojas et al, 2018). Estas situaciones implican las limitaciones para la presencia de la especie endémica E. mutisii en las cuencas de la jurisdicción CAR, evidenciando una disminución continua tanto de su calidad del agua como de sus hábitats asociados (CAR, 2016a). Esto se hizo manifiesto en los ríos Ubaté y Suárez, que además de recoger los vertimientos de varios municipios sufren continuamente de obras de adecuación hidráulica, como dragados y limpieza de cauces (CAR, 2018).

La relación peso-talla que ya había sido reportada para poblaciones del pez capitán (Bastidas y Lemus, 2014), muestra que los incrementos de peso fueron más rápidos que los incrementos en tallas (Gómez, 2014).

A su vez, se evidenció una marcada heterogeneidad de tallas y pesos en los ejemplares capturados de E. mutisii, que implica la existencia de diferentes condiciones de hábitat reunidos en los cuerpos hídricos evaluados en Cundinamarca que favorecen la presencia del pez capitán en diferentes estados de su ciclo de vida, como rangos altitudinales óptimos, así como de temperatura y concentración de oxígeno adecuadas. No obstante, se encontró un número de alevines muy bajo (solamente un ejemplar en el embalse Tominé), debido a la pérdida de refugios y otras amenazas que enfrentan en general los cuerpos hídricos en el departamento. Todas estas amenazas vienen dadas por diferentes actividades antrópicas, como los vertimientos y las obras de adecuación hidráulica de los cauces, ejecutadas por la autoridad ambiental del departamento (CAR, 2009b, 2016a).

\section{CONCLUSIONES}

Aunque E. mutisii se registra en la mayoría de los cuerpos lénticos de Cundinamarca, en altitudes altas, el número de individuos recolectados fue menor en comparación con registros históricos de capturas. Esta situación también se presentó en ecosistemas lóticos, siendo más atenuado en lagunas y embalses que mantienen las poblaciones más estables para la especie, aún con el incremento actual de vertimientos 
y el aumento de impactos provocados por actividades antrópicas. Sin embargo, la especie, que se encuentra categorizada como vulnerable, podría subir en la escala de amenaza aún en estos cuerpos lénticos.

En efecto, la especie enfrenta la situación más drástica en los ecosistemas lóticos del departamento de Cundinamarca, ya que las condiciones ambientales de los ríos no logran compensar los efectos generados por las actividades antrópicas. Por ejemplo, para los ríos Bogotá, Frío, Ubaté y Veraguas que, en sus cuencas altas presentan una calidad del agua óptima, no fue posible reportar la presencia del pez capitán en este estudio. Razón por la cual se hace imperativo ejecutar el plan de manejo propuesto para la conservación de la especie en la jurisdicción CAR (Rojas et al, 2018), así como generar el repoblamiento del pez capitán en las zonas dónde se supone una disminución sustancial de su abundancia poblacional. Por lo tanto, es importante reevaluar la categoría de amenaza actual de E. mutisii pues se constató que el grado de deterioro es significativamente alto, lo que disminuye considerablemente la densidad de individuos en estado alevín.

Asimismo, el protocolo metodológico utilizado en esta investigación puede ser aplicado en otros cuerpos de agua pertenecientes al área de distribución natural de la especie, en el altiplano cundiboyacense, que hacen parte de la jurisdicción de la CAR Cundinamarca y la Corporación Autónoma Regional de Boyacá, con miras a la obtención de información sobre la composición y abundancia íctica, ya que cumple con el rigor científico requerido para dicho fin.

\section{AGRADECIMIENTOS}

Este estudio hace parte del Convenio 1835 celebrado y financiado entre la Universidad Manuela Beltrán (UMB) y la Corporación Autónoma Regional de Cundinamarca (CAR). Los autores quieren expresar su especial agradecimiento a las directivas de la UMB y la CAR por todo el apoyo recibido. Igualmente, al funcionario de la CAR Nelson Londoño Gutiérrez y Juan Camilo Rey; a Vivian Bojacá Bautista, Laura Díaz Ospina y Laura López Cubides por la interventoría al convenio; al grupo de ingenieros ambientales y jóvenes investigadores: Anderson Bermúdez
Barrantes, Omar Carreño Puentes, Catalina Igua Cortés, Paola Pineda Suárez y Hernando Baquero Gamboa.

\section{CONFLICTO DE INTERESES}

Los autores declaran no tener conflictos de intereses.

\section{REFERENCIAS}

Arias-Pineda, J.Y., y Pedroza-Martínez, D.R. (2018). Presencia del cangrejo rojo Procambarus clarkii (Girard, 1852) en la sabana de Bogotá, Colombia. Boletín de la Sociedad Entomológica Aragonesa, 62, 283-286. Recuperado de http://sea-entomologia.org/Publicaciones/Boletines/ boletinSEA.htm

Baptiste, M.P., Castaño, N., Cárdenas, D., Gutiérrez, F.P., Gil, D, y Lasso, C.A. (Eds.). (2010). Análisis de riesgo y propuesta de categorización de especies introducidas para Colombia. Bogotá: Instituto de Investigación en Recursos Biológicos Alexander von Humboldt. Recuperado de http://hdl.handle.net/20.500.11761/31384

Bastidas, J., y Lemus, C. (2012). Aspectos bioecológicos del capitán de la sabana Eremophilus mutisii Humboldt 1805 (Pisces: Trichomycteridae) en el altiplano cundiboyacense. Bogotá: Universidad Manuela Beltrán.

Bastidas, J., y Lemus, C. (2014). Aporte al conocimiento de la biología y ecología del pez capitán de la sabana (Eremophilus mutisii) Humboldt 1805 (Pisces: Trichomycteridae) en el altiplano cundiboyacense. Bogotá: Corporación Universitaria de Ciencia y Desarrollo.

Caicedo, C. (2017). Análisis del declive de la especie Eremophilus mutisii. (Trabajo de Grado). Bogotá: Escuela de Ciencias Agrícolas, Pecuarias y del Medio Ambiente Universidad Nacional Abierta y a Distancia.

Corporación Autónoma Regional de Cundinamarca - CAR. (2009a). Resolución 3460 del 28 de diciembre de 2009 por la cual se establecen los objetivos de calidad del agua para la cuenca del río Negro, a lograr en el año 2020. Bogotá: CAR. Recuperado de https://www.car.gov.co/uploads/ files/5ada1004bb1a0.pdf

Corporación Autónoma Regional de Cundinamarca - CAR. (2009b). Resolución 3462 del 28 de diciembre de 2009 por la cual se establecen los objetivos de calidad del agua para la cuenca de los ríos Ubaté y Suárez, a lograr en el año 2020. Bogotá: CAR. Recuperado de https: //www.car.gov.co/uploads/files/5ac79c5b18c9e.pdf

Corporación Autónoma Regional de Cundinamarca - CAR. (2009c). Adecuación Hidráulica y Recuperación Ambiental del Río Bogotá: Evaluación Ambiental y Plan de Gestión Ambiental. Volumen II. Evaluación Ambiental. Bogotá: CAR. Recuperado de http://web2.car.gov.co/EvaluacionAmbientalVolumenII .pdf

Corporación Autónoma Regional de Cundinamarca - CAR. (2016a). Resolución 0136 del 26 de enero de 2016 por 
medio de la cual se adopta la Guía técnica para la adecuación hidráulica y restauración ambiental de corrientes hídricas superficiales en la jurisdicción de la Corporación Autónoma Regional de Cundinamarca. Bogotá: CAR. Recuperado de https://www.car.gov.co/uploads/ files/5af083bab4a24.pdf

Corporación Autónoma Regional de Cundinamarca - CAR. (2016b). Plan de Manejo y Control del Cangrejo Rojo Americano (Procambarus clarkii) en la jurisdicción $C A R$. Bogotá: Dirección de Modelamiento, Monitoreo y Laboratorio Ambiental CAR. Recuperado de https: //www.car.gov.co/uploads/files/5b7c66651ce85.pdf

Corporación Autónoma Regional de Cundinamarca - CAR. (2018). Estado del recurso hídrico en la cuenca del río medio y Bajo Suárez en términos de calidad y cantidad. Bogotá: Dirección de Recursos Naturales CAR. Recuperado de https://www.car.gov.co/uploads/files/5bae43fc52af5. pdf

Correia, A.M. (2002). Niche breadth and trophic diversity: feeding behavior of the red swamp crayfish (Procambarus clarkii) towards environmental availability of aquatic macroinvertebrates in a rice field (Portugal). Acta Oecologica, 23(6), 421-429. Recuperado de https://www.sciencedirect.com/science/article/abs/ pii/S1146609X02011669

Correia, A.M., Bandeira, N., y Anastácio, P.M. (2007). Influence of chemical and visual stimuli in food-search behaviour of Procambarus clarkii under clear conditions. Marine and Freshwater Behaviour and Physiology, 40(3), 189194. doi:10.1080/10236240701600725

Dahl, G. (1971). Los peces del norte de Colombia. Bogotá: Instituto de Desarrollo de los Recursos Naturales Renovables INDERENA, Ministerio de Agricultura.

DoNascimiento, C, Prada-Pedreros, S., y Guerrero-Kommritz, J. (2014). Trichomycterus venulosus (Steindachner, 1915), a junior synonym of Eremophilus mutisii Humboldt, 1805 (Siluformes: Trichomycteridae) and not an extinct species. Neotropical Ichthyology, 12(4), 707-715. doi:10.1590/1982-0224-20130236

Elosegi, A., y Sabater, S. (Eds.). (2009). Conceptos y técnicas en ecología fluvial. Madrid, España: Fundación BBVA. Recuperado de https://www.fbbva.es/wp-content/uploads/2017/ 05/dat/DE_2009_conceptos_ecologia_fluvial.pdf

Elvira, B., y Almodóvar, A. (2010). La suelta de truchas alóctonas es muy perjudicial para el ecosistema. Trofeo Pesca, 172, 102-103. Recuperado de https://www.ucm. es/data/cont/docs/568-2013-12-15-172-2010

Espinosa-Pérez, H. (2014). Biodiversidad de peces en México. Revista Mexicana de Biodiversidad, 85, 450-459. doi:10.7550/rmb.32264

Galván-Guevara, S., y De la Ossa, V.J. (2011). Fauna exótica y fauna trasplantada con mayor representatividad en Colombia. Revista Colombiana de Ciencia Animal, 3(1), 167-179. doi:10.24188/recia.v3.n1.2011.358

García-Berthou, E., Almeida, D., Benejam, L., Magellan, K., Bae, M.J., Casals, F., y Merciai, R. (2015). Impacto ecológico de los peces continentales introducidos en la península ibérica. Ecosistemas, 24(1), 36-42. doi:10.7818/ECOS.2015.24-1.07
Ginés, E. (2011). Pesca eléctrica sin muerte para conocer las poblaciones piscícolas. Natural de Aragón. Revista Trimestral del Departamento de Medio Ambiente del Gobierno de Aragón, 43, 17. Recuperado de http://bibliotecavirtual.aragon.es/bva/i18n/ catalogo_imagenes/grupo.cmd?path $=3705669$

Gómez, S. (2014). Las relaciones longitud peso en algunos peces tropicales de acuario. Revista Científica Internacional de Acuicultura Española - AquaTIC, 41, 1-7. Recuperado de http://www.revistaaquatic.com/ojs/index.php/ aquatic/article/view/57/48

Gutiérrez-Bonilla, F.D.P., y Álvarez-León, R. (2011). Los cíclidos (Pisces: Cichlidae) en Colombia: introducciones, trasplantes y repoblaciones. Revista Luna Azul, 33, 154-177. Recuperado de http://www.scielo.org.co/scielo. php?script=sci_arttext\&pid=S1909-24742011000200013

Huxley, J.S, y Teisser, G. (1936). Terminology of relative growth. Nature, 137, 780-781. doi:10.1038/137780b0

Jacobsen, D., y Dangles, O. (2017). Ecology of high-altitude waters. Oxford, United Kingdom: Oxford University Press.

Kjelland, M.E., Woodley, C.M., Swannack, T.M., y Smith, D.L. (2015). A review of the potential effects of suspended sediment on fishes: potential dredging-related physiological, behavioral, and transgenerational implications. Environment Systems and Decisions, 35(3), 334350. doi:10.1007/s10669-015-9557-2

Lemus-Portillo, C., Echavarría, M., Avella, C., López, W., Useche, P., y Mojica, I. (2017). Programa nacional para la conservación de la especie endémica de Colombia pez capitán de la sabana (Eremophilus mutisii). Bogotá: Ministerio de Ambiente y Desarrollo Sostenible - Universidad Manuela Beltrán.

Lobón-Cerviá, J. (1991). Dinámica de poblaciones de peces en ríos: pesca eléctrica y métodos de capturas sucesivas en la estima de abundancias. Madrid, España: Centro de Investigaciones del agua. Recuperado de http: //www.scielo.org.co/scielo.php?script=sci_nlinks\&ref= 000083\&pid=S0122-7483201300010000500026\&lng=en

Maldonado-Ocampo, J.A., Vari, R.P., y Usma, J.S. (2008). Checklist of the freshwater fishes of Colombia. Biota colombiana, 9(2), 143-237. Recuperado de https://www. redalyc.org/articulo.oa?id $=49120960001$

Mayorga, M. (1992). Biología reproductiva y alimentación de las poblaciones del capitán de la sabana, Eremophilus mutisii, en la laguna de Fúquene. (Trabajo de Grado). Bogotá: Biología Marina, Universidad Jorge Tadeo Lozano.

Miles, C. (1942). Descripción sistemática del "Pez Graso" del Lago de Tota (Boyacá). Caldasia, 5, 55-58. Recuperado de https://revistas.unal.edu.co/index.php/cal/ article/view/31796/31826

Mojica, J.I., Castellanos, C., Usma, J.S., y Álvarez-León, R. (Eds.). (2002). Libro Rojo de Peces dulceacuícolas de Colombia 2002. Serie Libros Rojos de Especies Amenazadas de Colombia. Bogotá: Instituto de Ciencias Naturales, Universidad Nacional de Colombia, Ministerio del Medio Ambiente. Recuperado de https://www.academia.edu/15487005/Libro_rojo_de_ peces_dulceacuícolas_de_Colombia

Mojica, J.I., Usma, J.S., Álvarez-León, R., y Lasso, C.A. (Eds.). (2012). Libro rojo de peces dulceacuí- 
colas de Colombia 2012. Serie Libros Rojos de Especies Amenazadas de Colombia. Bogotá: Instituto de Investigación de Recursos Biológicos Alexander von Humboldt, Instituto de Ciencias Naturales de la Universidad Nacional de Colombia, WWF Colombia y la Universidad de Manizales. Recuperado de http://awsassets.panda.org/downloads/libro_rojo_ peces_dulceacuicolas_de_colombia__dic_2012.pdf

Narváez-Barandica, J.C., Herrera-Pertuz, F.A., y BlancoRacedo, J. (2008). Efecto de los artes de pesca sobre el tamaño de los peces en una pesquería artesanal del Caribe colombiano. Boletín de Investigaciones Marinas y Costeras-INVEMAR, 37(2), 163-187. Recuperado de http://www.scielo.org.co/pdf/mar/v37n2/v37n2a09.pdf

Pachón, Y., y Valderrama, M. (2018). Anotaciones al estado, uso y gestión de la langostilla roja Procambarus (Scapulicambarus) clarkii, especie invasora en la laguna de Fúquene (Cundinamarca, Colombia). Biodiversidad en la Práctica, Documentos de trabajo del Instituto Humboldt, 3(1), 30-51. Recuperado de http://revistas.humboldt. org.co/index.php/BEP/article/view/544/511

Parasiewicz, P., Gortazar, J., Sánchez, M.M., y García de Jalón, D. (2009). MesoHABSIM: An effective tool for river and watershed management. Tecnología y Ciencias del Agua, 29(309), 20-26. Recuperado de https://www. researchgate.net/publication/287164797_MesoHABSIM_ An_effective_tool_for_river_and_watershed_management

Prada-Pedreros, S., Rivera-Rondón, C.A., y GuerreroKommritz, J. (2006). Trichomycterus venulosus (Steindachner, 1915): posible extint species from the páramo de Cruz Verde (Cundinamarca, Colombia). Biota Colombiana, 7(1), 163-166. Recuperado de https://www.redalyc. org/articulo.oa?id=49170111

Pulido-Méndez, O.L. (2013). Influencia del hábitat físico sobre la ictiofauna-cuenca baja río Dagua: caso de la quebrada Jesús. (Tesis de Maestría). Palmira, Valle: Universidad Nacional de Colombia. doi:10.13140/RG.2.2.36050.35527

Ricker, W.E. (1975). Computation and interpretation of biological statistics of fish populations. Bulletin Fisheries Research Board of Canada, 191, 1-382. doi:10.2307/3800109

Rodríguez-Forero, A. (2000). El pez "Capitán de la Sabana", Eremophilus mutisii, en el altiplano cundiboyacense. Colombia. Ciencia y Tecnología, 18(2), 38-40. http://hdl. handle.net/11146/2181

Rojas, J.E., Echavarria, M., y Lemus-Portillo, C. (2018). Plan de manejo y conservación de la especie (Eremophilus mutisii) para la jurisdicción CAR. Bogotá: Corporación Autónoma Regional de Cundinamarca y Universidad Manuela Beltrán.

Roldán, G., y Ramírez, J.J. (2008). Fundamentos de limnología neotropical ( $2^{a}$ ed.). Medellín: Universidad de Antioquia.

Rosado, R., y González, J.A. (2007). Compilación bibliográfica y análisis del estado actual de la investigación sobre capitán de la sabana, Eremophilus mutisii. Revista de la Asociación Colombiana de Ictiólogos - Dahlia, 9, 43-51. Recuperado de https://www.acictios.org/dahlia/ Revista-Dahlia-9.pdf

Smokorowski, K.E., y Pratt, T.C. (2007). Effect of a change in physical structure and cover on fish and fish habitat in freshwater ecosystems-a review and meta-analysis. Envi- ronmental Reviews, 15(NA), 15-41. doi:10.1139/A06-007

Sostoa, A., García de Jalón, D., y García, E. (2005). Metodología para el establecimiento del estado ecológico según la Directiva MARCO del agua: protocolos de muestreo y análisis para ictiofauna. Madrid: Ministerio de Medio Ambiente, Confederación Hidrográfica del Ebro. Recuperado de http://195.55.247.234/webcalidad/estudios/ indicadoresbiologicos/Manual_ictiofauna.pdf

Vezza, P., Parasiewicz, P., Spairani, M., y Comoglio, C. (2014). Habitat modeling in highgradient streams: the mesoscale approach and application. Ecological Applications, 24(4), 844-861. doi:10.1890/11-2066.1 